\title{
Bordered Constructions of Self-Dual Codes from Group Rings and New Extremal Binary Self-Dual Codes
}

\author{
Steven T. Dougherty \\ Department of Mathematics \\ University of Scranton \\ Scranton, PA 18510, USA \\ Joseph Gildea, Adrian Korban \\ University of Chester \\ Department of Mathematics \\ Chester, UK \\ Abidin Kaya \\ Sampoerna Academy, L'Avenue Campus \\ 12780, Jakarta, Indonesia \\ Alexander Tylyshchak \\ Department of Algebra \\ Uzhgorod State University \\ Uzhgorod, Ukraine \\ Bahattin Yildiz* \\ Department of Mathematics \& Statistics \\ Northern Arizona University \\ Flagstaff, AZ 86001,USA \\ bahattin.yildiz@nau.edu
}

\begin{abstract}
We introduce a bordered construction over group rings for self-dual codes. We apply the constructions over the binary field and the ring $\mathbb{F}_{2}+u \mathbb{F}_{2}$, using groups of
\end{abstract}

*corresponding author 
orders $9,15,21,25,27,33$ and 35 to find extremal binary self-dual codes of lengths $20,32,40,44,52,56,64,68,88$ and best known binary self-dual codes of length 72 . In particular we obtain 41 new binary extremal self-dual codes of length 68 from groups of orders 15 and 33 using neighboring and extensions. All the numerical results are tabulated throughout the paper.

Key Words: Group rings; self-dual codes; codes over rings; extremal codes; bordered constructions

\section{Introduction}

A classical technique for producing self-dual codes over fields and rings has been to consider generator matrices of the form $\left(I_{n} \mid A\right)$ where $A$ is a circulant matrix satisfying $A A^{T}=-I_{n}$. This technique is naturally extended to the bordered construction where the matrix $A$ is replaced with

$$
\left(\begin{array}{c|ccc}
\gamma & \alpha & \ldots & \alpha \\
\hline \alpha & & & \\
\vdots & & A & \\
\alpha & & &
\end{array}\right)
$$

Both methods have been used extensively in the literature to produce many extremal binary self-dual codes. (See [18], [22], [14] and references therein for some related works.)

Using group rings in coding theory has introduced new tools in constructing codes. A classical way to produce a code from a group ring is to use the matrix representation of elements in a group ring and using zero divisors ([16], [17]). In [6], the idea was extended to any group $G$ and $G$-codes were defined as codes that are ideals in the group $\operatorname{ring} R G$ where $R$ is a finite Frobenius ring. Group rings have also been used in constructing extremal binary self-dual codes. In $[1,19]$ and [20], the extended binary Golay code and the extended Quadratic residue code were constructed from group rings using the symmetric group of degree four and dihedral groups. There have been more recent works in which the construction is generalized and modified to include many more groups and self-dual codes of different lengths. The main idea in the most recent works is to use a matrix of the form $\left[I_{n} \mid A\right]$, where $A$ is the image of a unitary unit in a group ring under a map that sends group ring elements to matrices. This idea has been successfully used in producing extremal binary self-dual codes in [12] and [13].

In this work, we will modify the constructions by introducing a border to $I_{n}$ as well as to the matrix $A$ used in the group ring constructions found in [12] and [13]. Using groups of orders, 9, 15, 21, 25, 27, 33 and 35 over the binary field or the ring $R_{1}=\mathbb{F}_{2}+u \mathbb{F}_{2}$, we construct a considerable number of extremal binary self-dual codes of lengths 20, 32, 40, $44,52,56,64,68,88$ and best known binary self-dual codes of length 72 . In particular we 
construct 41 extremal binary self-dual codes of length 68 with new weight enumerators from groups of orders 15 and 33, using neighboring and extension methods in addition to the bordered group ring constructions.

The rest of the work is organized as follows. In Section 2, we give the preliminaries on group rings, self-dual codes and the alphabets that we use. In Section 3, we give the bordered group ring construction and prove the main results. In Section 4, we apply the constructions over different groups to find extremal binary self-dual codes of different lengths that we enumerate in tables. In section 5, we focus on the new extremal binary self-dual codes of length 68 that we obtain from groups of order 15 and 33. Section 6 ends the paper with concluding remarks and directions for possible future directions.

\section{Preliminaries}

\section{$2.1 \quad$ Codes}

In this paper, we will consider two main alphabets, the finite field $\mathbb{F}_{2}$ and the ring $\mathbb{F}_{2}+u \mathbb{F}_{2}$. Both alphabets are special examples of a larger class of rings called Frobenius rings. A code over a finite commutative ring $R$ is defined as any subset $C$ of $R^{n}$. When the code is a submodule of the ambient space then the code is said to be linear. To the ambient space, we

attach the usual inner-product, specifically $[\mathbf{v}, \mathbf{w}]=\sum v_{i} w_{i}$. The orthogonal with respect to this inner-product is defined as $C^{\perp}=\left\{\mathbf{w} \mid \mathbf{w} \in R^{n},[\mathbf{w}, \mathbf{v}]=0, \forall \mathbf{v} \in C\right\}$. Over Frobenius rings, we have that for all linear codes over $R,|C|\left|C^{\perp}\right|=|R|^{n}$. The proof of this fact and a complete description of codes over rings can be found in [5].

If a code satisfies $C=C^{\perp}$ then the code $C$ is said to be self-dual. If $C \subseteq C^{\perp}$ then the code is said to be self-orthogonal. For binary codes, a self-dual code where all weights are congruent to $0(\bmod 4)$ is said to be Type II and the code is said to be Type I otherwise. Two self dual codes $C$ and $D$ of length $n$ are said to be neighbours if $\operatorname{dim}(C \cap D)=n / 2-1$.

The Hamming weight enumerator of a code is defined as:

$$
W_{C}(x, y)=\sum_{\mathbf{c} \in C} x^{n-w t(\mathbf{c})} y^{w t(\mathbf{c})}
$$

where $w t(\mathbf{c})$ is the Hamming weight of the codeword $\mathbf{c}$. This is the homogeneous version of the weight enumerator. Extremal binary self-dual codes, which make up an important part of our work have certain weight enumerators, which are usually expressed in one variable, which is simply obtained by replacing $x$ by 1 in the above expression.

An upper bound on the minimum Hamming distance of a binary self-dual code finalized in $[21]$.

Theorem 2.1. ([21]) Let $d_{I}(n)$ and $d_{I I}(n)$ be the minimum distance of a Type I and Type 
II binary code of length $n$, respectively. Then

$$
d_{I I}(n) \leq 4\left\lfloor\frac{n}{24}\right\rfloor+4
$$

and

$$
d_{I}(n) \leq\left\{\begin{array}{lll}
4\left\lfloor\frac{n}{24}\right\rfloor+4 & \text { if } n \not \equiv 22 \quad(\bmod 24) \\
4\left\lfloor\frac{n}{24}\right\rfloor+6 & \text { if } n \equiv 22 \quad(\bmod 24)
\end{array}\right.
$$

Self-dual codes meeting these bounds are called extremal. Throughout the paper, we will be constructing extremal binary self-dual codes of different lengths.

\subsection{Group Rings}

We shall use group rings in our construction, so we give the standard definition of a group ring. Let $G$ be a finite group of order $n$, then the group ring $R G$ consists of $\sum_{i=1}^{n} \alpha_{i} g_{i}$, $\alpha_{i} \in R, g_{i} \in G$.

Addition in the group ring is done by coordinate addition, namely

$$
\sum_{i=1}^{n} \alpha_{i} g_{i}+\sum_{i=1}^{n} \beta_{i} g_{i}=\sum_{i=1}^{n}\left(\alpha_{i}+\beta_{i}\right) g_{i}
$$

The product of two elements in a group ring is given by

$$
\left(\sum_{i=1}^{n} \alpha_{i} g_{i}\right)\left(\sum_{j=1}^{n} \beta_{j} g_{j}\right)=\sum_{i, j} \alpha_{i} \beta_{j} g_{i} g_{j} .
$$

It follows that the coefficient of $g_{k}$ in the product is $\sum_{g_{i} g_{j}=g_{k}} \alpha_{i} \beta_{j}$.

We restrict ourselves to finite groups since we are only concerned with using these to construct codes but group rings can be defined for groups of arbitrary cardinality. Throughout, we shall use $e_{G}$ to denote to the identity element of any group $G$.

The following construction of a matrix was first given for codes over fields by Hurley in [16]. Let $R$ be a finite commutative Frobenius ring and let $G=\left\{g_{1}, g_{2}, \ldots, g_{n}\right\}$ be a group of order $n$. Let $v=\alpha_{g_{1}} g_{1}+\alpha_{g_{2}} g_{2}+\cdots+\alpha_{g_{n}} g_{n} \in R G$. Define the matrix $\sigma(v) \in M_{n}(R)$ to be

$$
\sigma(v)=\left(\begin{array}{ccccc}
\alpha_{g_{1}^{-1} g_{1}} & \alpha_{g_{1}^{-1} g_{2}} & \alpha_{g_{1}^{-1} g_{3}} & \ldots & \alpha_{g_{1}^{-1} g_{n}} \\
\alpha_{g_{2}^{-1} g_{1}} & \alpha_{g_{2}^{-1} g_{2}} & \alpha_{g_{2}^{-1} g_{3}} & \ldots & \alpha_{g_{2}^{-1} g_{n}} \\
\vdots & \vdots & \vdots & \vdots & \vdots \\
\alpha_{g_{n}^{-1} g_{1}} & \alpha_{g_{n}^{-1} g_{2}} & \alpha_{g_{n}^{-1} g_{3}} & \ldots & \alpha_{g_{n}^{-1} g_{n}}
\end{array}\right) .
$$

We note that the elements $g_{1}^{-1}, g_{2}^{-1}, \ldots, g_{n}^{-1}$ are the elements of the group $G$ in a some given order. We begin with a technical lemma.

Lemma 2.2. If $v=\sum_{i=1}^{n} \alpha_{g_{i}} g_{i}$ is a unitary unit of $R G$ and $\mu=\sum_{i=1}^{n} \alpha_{g_{i}}$ then $\mu^{2}=1$. 
Proof. The map $*: R G \longrightarrow R G$ defined by $\left(\sum_{g \in G} a_{g} g\right)^{*}=\sum_{g \in G} a_{g} g^{-1}$ is an antiautomorphism of $R G$ of order 2. An element $v$ of $V(K G)$ satisfying $v v^{*}=1$ is called unitary. The homomorphism $\varepsilon: R G \longrightarrow R$ given by $\varepsilon\left(\sum_{i=1}^{n} \alpha_{g_{i}} g_{i}\right)=\sum_{i=1}^{n} \alpha_{g_{i}}$ is called the augmentation mapping of $R G$. Let $v=\sum_{i=1}^{n} \alpha_{g_{i}} g_{i}$, then $v^{*}=\sum_{i=1}^{n} \alpha_{g_{i}} g_{i}^{-1}$ and $\varepsilon(v)=\varepsilon\left(v^{*}\right)=\sum_{i=1}^{n} \alpha_{g_{i}}=$ $\mu$. Therefore $\varepsilon\left(v v^{*}\right)=\varepsilon(v) \varepsilon\left(v^{*}\right)=\mu^{2}=1$.

\section{$2.3 \quad$ A Family of Rings}

We shall use extensively a family of rings of characteristic 2. These were defined in [10] and [11].

For $k \geq 1$, define the commutative ring

$$
R_{k}=\mathbb{F}_{2}\left[u_{1}, u_{2}, \ldots, u_{k}\right] /\left\langle u_{1}^{2}, u_{2}^{2}, \ldots, u_{k}^{2}\right\rangle, \quad u_{i} u_{j}=u_{j} u_{i}, \quad 1 \leq i \neq j \leq k .
$$

We can also define the rings recursively as:

$$
R_{k}=R_{k-1}\left[u_{k}\right] /\left\langle u_{k}^{2}\right\rangle=R_{k-1}+u_{k} R_{k-1}
$$

For any subset $A \subseteq\{1,2, \ldots, k\}$ we will fix

$$
u_{A}:=\prod_{i \in A} u_{i}
$$

with the convention that $u_{\emptyset}=1$. Then any element of $R_{k}$ can be represented as

$$
\sum_{A \subseteq\{1, \ldots, k\}} c_{A} u_{A}, \quad c_{A} \in \mathbb{F}_{2} .
$$

An advantage of representing elements with this notation is that we can easily observe that

$$
u_{A} u_{B}= \begin{cases}0 & \text { if } A \cap B \neq \emptyset \\ u_{A \cup B} & \text { if } A \cap B=\emptyset .\end{cases}
$$

This leads to

$$
\left(\sum_{A} c_{A} u_{A}\right)\left(\sum_{B} c_{B} u_{B}\right)=\sum_{A, B \subseteq\{1, \ldots, k\}, A \cap B=\emptyset} c_{A} c_{B} u_{A \cup B}
$$

It is shown in [10] that the ring $R_{k}$ is a commutative ring with $\left|R_{k}\right|=2^{\left(2^{k}\right)}$.

The following lemma, which has also been proven in [10], will be used in subsequent sections.

Lemma 2.3. An element $\gamma$ of $R_{k}$ that is a unit satisfies $\gamma^{2}=1$. An element $\alpha$ of $R_{k}$ that is a non-unit satisfies $\alpha^{2}=0$. 
The Gray map from $R_{k}$ to $\mathbb{F}_{2}^{2^{k}}$ is defined recursively, by building up from the smaller cases. For $R_{1}$ we have the following map from [9]:

$$
\phi_{1}\left(a+b u_{1}\right)=(b, a+b) .
$$

Then let $c \in R_{k}, c$ can be written as $c=a+b u_{k-1}, a, b \in R_{k-1}$. Then

$$
\phi_{k}(c)=\left(\phi_{k-1}(b), \phi_{k-1}(a+b)\right) .
$$

The map $\phi_{k}$ is a distance preserving map and the following is shown in [11].

Theorem 2.4. Let $C$ be a self-dual code over $R_{k}$, then $\phi_{k}\left(R_{k}\right)$ is a binary self-dual code of length $2^{k} n$.

The next result proves very useful when extending codes over $R_{k}$. It is proven in [7] but we state it in terms relevant to this paper.

Theorem 2.5. Let $\mathcal{C}$ be a self-dual code over $R_{k}$ of length $n$ and $G=\left(r_{i}\right)$ be a $j \times n$ generator matrix for $\mathcal{C}$, where $r_{i}$ is the $i$-th row of $G, 1 \leq i \leq k$. Let $c$ be a unit in $R_{k}$ and $X$ be a vector in $R_{k}{ }^{n}$ with $\langle X, X\rangle=1$. Let $y_{i}=\left\langle r_{i}, X\right\rangle$ for $1 \leq i \leq k$. Then the following matrix

$$
\left(\begin{array}{cc|c}
1 & 0 & X \\
\hline y_{1} & c y_{1} & r_{1} \\
\vdots & \vdots & \vdots \\
y_{k} & c y_{k} & r_{k}
\end{array}\right)
$$

generates a self-dual code $\mathcal{C}^{\prime}$ over $R_{k}$ of length $n+2$.

We note that since $c$ is a unit in $R_{k}$ it necessarily satisfies $c^{2}=1$. In [7] this condition is an added condition but we can eliminate it over $R_{k}$.

Remark 1. In the subsequent parts of the paper, we will mainly use the special cases $\mathbb{F}_{2}=R_{0}$ and $R_{1}=\mathbb{F}_{2}+u \mathbb{F}_{2}$ for our constructions. However, it is to be noted that the theoretical results are valid for all $R_{k}$.

\section{The bordered group ring construction}

Let $v \in R G$ where $R$ is a finite Frobenius ring of characteristic 2 and $G$ be a finite group of order $n$. Define the following matrix:

$$
M(\sigma)=\left(\begin{array}{c|ccc|c|ccc}
\gamma_{1} & \alpha_{1} & \cdots & \alpha_{1} & \gamma_{2} & \alpha_{2} & \cdots & \alpha_{2} \\
\hline \alpha_{1} & & & & \alpha_{2} & & & \\
\vdots & & I_{n} & & \vdots & & \sigma(v) & \\
\alpha_{1} & & & & \alpha_{2} & &
\end{array}\right)
$$


where $\sigma(v)$ is the matrix associated to the element $v$ of the group ring, as given in 4 .

By $C_{\sigma}$ we denote the linear code generated by $M(\sigma)$ over the ambient alphabet.

We give the following sufficient conditions for $M(\sigma)$ to proiduce self-dual codes. Note that not all self-dual codes will be constructed in this way.

Theorem 3.1. Let $R$ be a finite commutative Frobenius ring of characteristic 2 and let $G=\left\{g_{1}, g_{2}, \ldots, g_{n}\right\}$ be a finite group of order $n$. If $\left(\gamma_{1}+\gamma_{2}\right)^{2}+n\left(\alpha_{1}+\alpha_{2}\right)^{2}=0$, vv $v^{*}=$ $1+\left(\alpha_{1}+\alpha_{2}\right)^{2} \widehat{g}$ and $\left(\gamma_{1}+n \alpha_{1}^{2}, \alpha_{1}+\mu \alpha_{2}, \gamma_{1}+n \alpha_{2}^{2}\right)$ has free rank 1 then $C_{\sigma}$ is a self-dual code of length $2 n+2$, where $\widehat{g}=\sum_{i=1}^{n} g_{i}$ and $\mu=\sum_{i=1}^{n} \alpha_{g_{i}-1}$.

Proof. Let $M(\sigma)=\left(\begin{array}{cccc}A_{1} & A_{2} & A_{3} & A_{4} \\ A_{2}^{T} & I_{n} & A_{4}^{T} & \sigma(v)\end{array}\right)$ where $A_{1}=\left(\gamma_{1}\right), A_{2}=\left(\begin{array}{lll}\alpha_{1} & \ldots & \alpha_{1}\end{array}\right), A_{3}=$ $\left(\gamma_{2}\right)$ and $A_{4}=\left(\begin{array}{lll}\alpha_{2} & \ldots & \alpha_{2}\end{array}\right)$. Then

$$
\begin{aligned}
M(\sigma) M(\sigma)^{T} & =\left(\begin{array}{cccc}
A_{1} & A_{2} & A_{3} & A_{4} \\
A_{2}^{T} & I_{n} & A_{4}^{T} & \sigma(v)
\end{array}\right)\left(\begin{array}{cc}
A_{1}^{T} & A_{2} \\
A_{2}^{T} & I_{n} \\
A_{3}^{T} & A_{4} \\
A_{4}^{T} & \sigma(v)^{T}
\end{array}\right) \\
& =\left(\begin{array}{cc}
A_{1} A_{1}^{T}+A_{2} A_{2}^{T}+A_{3} A_{3}^{T}+A_{4} A_{4}^{T} & A_{1} A_{2}+A_{2}+A_{3} A_{4}+A_{4} \sigma(v)^{T} \\
A_{2}^{T} A_{1}^{T}+A_{2}^{T}+A_{4}^{T} A_{3}^{T}+\sigma(v) A_{4}^{T} & A_{2}^{T} A_{2}+I_{n}+A_{4}^{T} A_{4}+\sigma(v) \sigma(v)^{T}
\end{array}\right) .
\end{aligned}
$$

Now,

$$
\begin{aligned}
A_{1} A_{1}^{T}+A_{2} A_{2}^{T}+A_{3} A_{3}^{T}+A_{4} A_{4}^{T} & =\gamma_{1}^{2}+\left(\begin{array}{lll}
\alpha_{1} & \ldots & \alpha_{1}
\end{array}\right)\left(\begin{array}{c}
\alpha_{1} \\
\vdots \\
\alpha_{1}
\end{array}\right)+\gamma_{2}^{2}+\left(\begin{array}{lll}
\alpha_{2} & \ldots & \alpha_{2}
\end{array}\right)\left(\begin{array}{c}
\alpha_{2} \\
\vdots \\
\alpha_{2}
\end{array}\right) \\
& =\gamma_{1}^{2}+n \alpha_{1}^{2}+\gamma_{2}^{2}+n \alpha_{2}^{2} \\
& =\left(\gamma_{1}+\gamma_{2}\right)^{2}+n\left(\alpha_{1}+\alpha_{2}\right)^{2}
\end{aligned}
$$

and

$$
\begin{aligned}
& A_{2}^{T} A_{2}+I_{n}+A_{4}^{T} A_{4}+\sigma(v) \sigma(v)^{T}=\left(\begin{array}{c}
\alpha_{1} \\
\vdots \\
\alpha_{1}
\end{array}\right)\left(\begin{array}{lll}
\alpha_{1} & \ldots & \alpha_{1}
\end{array}\right)+I_{n}+\left(\begin{array}{c}
\alpha_{2} \\
\vdots \\
\alpha_{2}
\end{array}\right)\left(\begin{array}{lll}
\alpha_{2} & \ldots & \alpha_{2}
\end{array}\right)+\sigma(v) \sigma\left(v^{*}\right) \\
& =\left(\begin{array}{ccc}
\alpha_{1}^{2} & \cdots & \alpha_{1}^{2} \\
\vdots & \ddots & \vdots \\
\alpha_{1}^{2} & \cdots & \alpha_{1}^{2}
\end{array}\right)+I_{n}+\left(\begin{array}{ccc}
\alpha_{2}^{2} & \cdots & \alpha_{2}^{2} \\
\vdots & \ddots & \vdots \\
\alpha_{2}^{2} & \cdots & \alpha_{2}^{2}
\end{array}\right)+\sigma\left(v v^{*}\right) \\
& =\left(\begin{array}{ccc}
\alpha_{1}^{2}+\alpha_{2}^{2} & \cdots & \alpha_{1}^{2}+\alpha_{2}^{2} \\
\vdots & \ddots & \vdots \\
\alpha_{1}^{2}+\alpha_{2}^{2} & \cdots & \alpha_{1}^{2}+\alpha_{2}^{2}
\end{array}\right)+I_{n}+\sigma\left(v v^{*}\right) .
\end{aligned}
$$


$C_{\sigma}$ is self-orthogonal since, $\operatorname{char}(R)=2$ and we have $\left(\gamma_{1}+\gamma_{2}\right)^{2}+n\left(\alpha_{1}+\alpha_{2}\right)^{2}=0$ and

$$
\sigma\left(v v^{*}\right)=I_{n}+\left(\begin{array}{ccc}
\alpha_{1}^{2}+\alpha_{2}^{2} & \cdots & \alpha_{1}^{2}+\alpha_{2}^{2} \\
\vdots & \ddots & \vdots \\
\alpha_{1}^{2}+\alpha_{2}^{2} & \cdots & \alpha_{1}^{2}+\alpha_{2}^{2}
\end{array}\right)=I_{n}+\left(\alpha_{1}+\alpha_{2}\right)^{2}\left(\begin{array}{ccc}
1 & \cdots & 1 \\
\vdots & \ddots & \vdots \\
1 & \cdots & 1
\end{array}\right)
$$

Moreover,

$$
\begin{aligned}
& \operatorname{rank}(M(\sigma))=\operatorname{rank}\left(\begin{array}{c|ccc|c|ccc}
\gamma_{1} & \alpha_{1} & \cdots & \alpha_{1} & \gamma_{2} & \alpha_{2} & \cdots & \alpha_{2} \\
\hline \alpha_{1} & & & & \alpha_{2} & & \\
\vdots & & I_{n} & & \vdots & & \sigma(v) & \\
\alpha_{1} & & & & \alpha_{2} & &
\end{array}\right) \\
& =\operatorname{rank}\left(\begin{array}{c|ccc|c|ccc}
\gamma_{1}+n \alpha_{1}^{2} & \alpha_{1} & \cdots & \alpha_{1} & \gamma_{2}+n \alpha_{2}^{2} & \alpha_{2} & \cdots & \alpha_{2} \\
\hline 0 & & & & 0 & & \\
\vdots & & I_{n} & \vdots & & \sigma(v) \\
0 & & & & 0 & &
\end{array}\right) \\
& =\operatorname{rank}\left[\left(\begin{array}{c|ccc|c|ccc}
\gamma_{1}+n \alpha_{1}^{2} & \alpha_{1} & \cdots & \alpha_{1} & \gamma_{2}+n \alpha_{2}^{2} & \alpha_{2} & \cdots & \alpha_{2} \\
\hline 0 & & & & 0 & & & \\
\vdots & & I_{n} & & \vdots & & \sigma(v) & \\
0 & & & & 0 & & &
\end{array}\right)\left(\begin{array}{cccc}
I & 0 & 0 & 0 \\
0 & I & 0 & 0 \\
0 & 0 & I & 0 \\
0 & \sigma(v)^{T} & 0 & I
\end{array}\right)\right]
\end{aligned}
$$




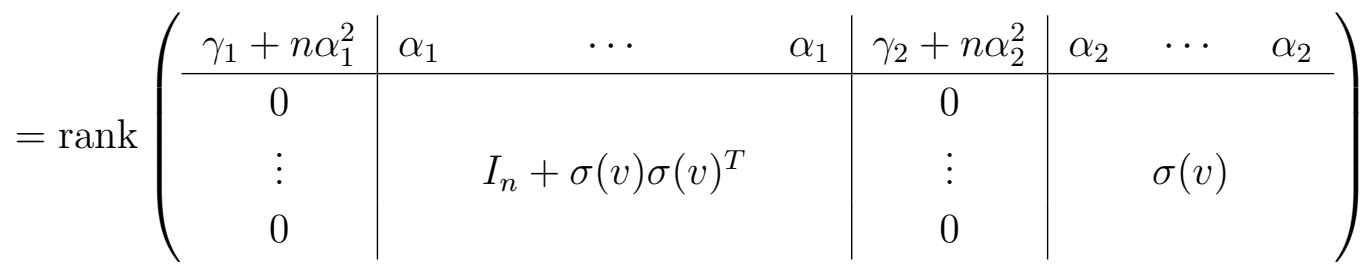

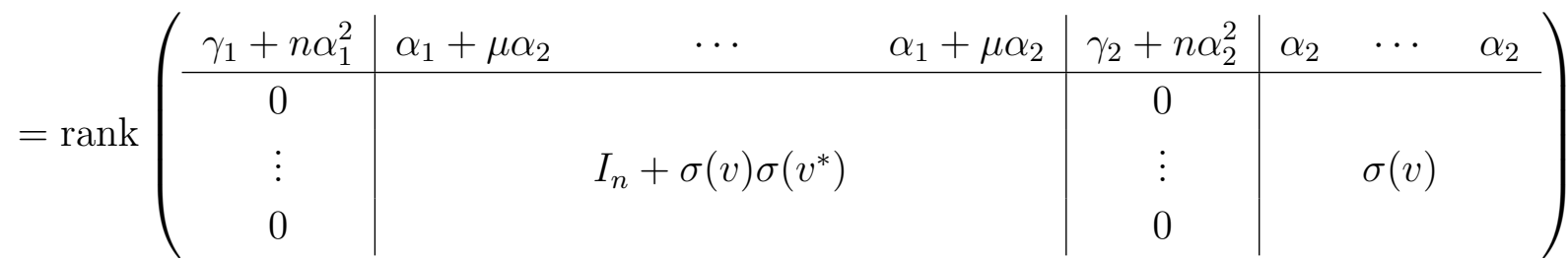

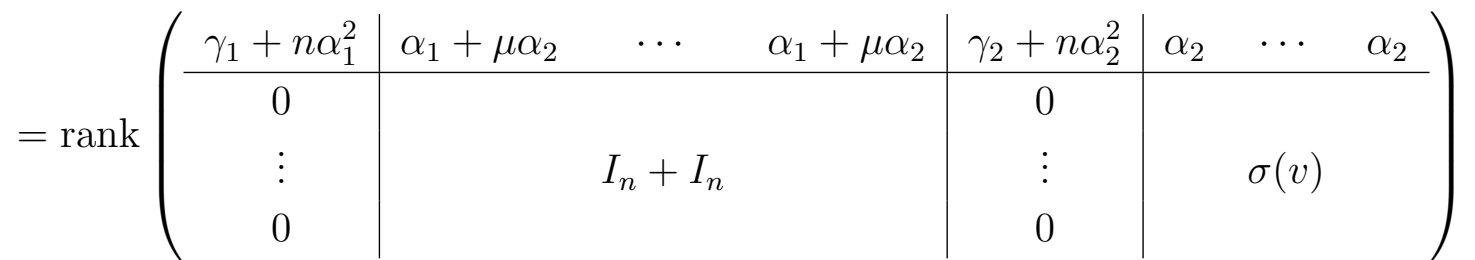

$$
\begin{aligned}
& =\operatorname{rank}\left(\begin{array}{c|ccc|c|ccc}
\gamma_{1}+n \alpha_{1}^{2} & \alpha_{1}+\mu \alpha_{2} & \cdots & \alpha_{1}+\mu \alpha_{2} & \gamma_{2}+n \alpha_{2}^{2} & \alpha_{2} & \cdots & \alpha_{2} \\
\hline 0 & & & & 0 & & \\
\vdots & & 0 & & \vdots & & \sigma(v) & \\
0 & & & & 0 & &
\end{array}\right) \\
& =\operatorname{rank}\left(\begin{array}{c|ccc|c|ccc}
\gamma_{1}+n \alpha_{1}^{2} & \alpha_{1}+\mu \alpha_{2} & \cdots & \alpha_{1}+\mu \alpha_{2} & \gamma_{2}+n \alpha_{2}^{2} & \tau & \cdots & \tau \\
\hline 0 & & & & 0 & & \\
\vdots & & 0_{n} & & \vdots & & \sigma(v) \\
0 & & & & 0 & &
\end{array}\right)
\end{aligned}
$$

where $\mu=\sum_{i=1}^{n} \alpha_{g_{i}{ }^{-1} g}$ and $\tau=\alpha_{2}+\mu^{2} \alpha_{2}=2 \alpha_{2}=0$ by Lemma 2.2. Therefore $C_{\sigma}$ is self-dual code if $\left(\gamma_{1}+n \alpha_{1}^{2}, \alpha_{1}+\mu \alpha_{2}, \gamma_{2}+n \alpha_{2}^{2}\right)$ has free rank 1, because then $M(\sigma)$ will have free rank $n+1$.

The family of rings $R_{k}$ is particularly well suited for this construction.

Corollary 3.2. Let $R=R_{k}$ and let $G$ be a finite group of order $n$. Let $v \in R G$ be a unitary unit. If $\alpha_{1}+\alpha_{2}$ is any non-unit then $C_{\sigma}$ is a self-dual code of length $2 n+2$.

Proof. We will show that this case satisfies the hypotheses of Theorem 3.1. If $v$ is a unitary unit then $v v^{*}=1$. If $\alpha_{1}+\alpha_{2}$ is a non-unit in $R_{k}$ then $\left(\alpha_{1}+\alpha_{2}\right)^{2}=0$ by Lemma 2.3. Thus $n\left(\alpha_{1}+\alpha_{2}\right)^{2}=n(0)=0$ and $v v^{*}=1$ satisfy the criteria in the Theorem.

\section{Numerical Results}

In what follows, we will give the computational results about binary self-dual codes obtained through the constructions described in Section 3, where the constructions are applied with 
groups of different orders. The search in these computations is done using the Magma Algebra System ([2]).

In many of the subsequent constructions, the matrix $\sigma(v)$ will have a special form, which is usually described in terms of circulant matrices or blocks. By $\operatorname{cir}\left(a_{1}, a_{2}, \ldots, a_{n}\right)$ we mean the $n \times n$ circulant matrix obtained by taking the vector $\left(a_{1}, a_{2}, \ldots, a_{n}\right)$ and all its cyclic shifts as rows.

$\operatorname{CIR}\left(A_{1}, A_{2}, \ldots, A_{n}\right)$ is very similar to $\operatorname{cir}\left(a_{1}, a_{2}, \ldots, a_{n}\right)$ in construction. The difference is that $A_{i}$ 's are $k \times k$ blocks instead of single elements. So, if each $A_{i}$ is a $k \times k$ block matrix, then $C I R\left(A_{1}, A_{2}, \ldots, A_{n}\right)$ is an $n k \times n k$ matrix. We used the different notation to distinguish between the single element case from the block case.

We will also use the concept of $g$-criculant matirces, which can be defined as follows: Let $0 \leq g \leq n$. A $g$-circulant matrix $B$ of order $n$ is a matrix of the form

$$
B=\operatorname{cir}_{g}\left(a_{1}, a_{2}, \ldots, a_{n}\right)=\left(\begin{array}{cccc}
a_{1} & a_{2} & \cdots & a_{n} \\
a_{n-g+1} & a_{n-g+2} & \cdots & a_{n-g} \\
a_{n-2 g+1} & a_{n-2 g+2} & \cdots & a_{n-2 g} \\
\vdots & \vdots & \ddots & \vdots \\
a_{g+1} & a_{g+2} & \cdots & a_{g}
\end{array}\right)
$$

where each subscript are calculated $\bmod n$

Note that, each row of $B$ is the previous row moved $g$ places to the right.

\subsection{Constructions from Groups of Order 9}

- Let $G=C_{9} \cong\left\langle x \mid x^{9}=1\right\rangle$. If $\alpha=\sum_{i=0}^{2} \sum_{j=0}^{2} a_{i+3 j+1} x^{3 i+j} \in R C_{9}$, then

$$
\sigma(\alpha)=\left(\begin{array}{ccc}
A_{1} & A_{2} & A_{3} \\
A_{3}^{\prime} & A_{1} & A_{2} \\
A_{2}^{\prime} & A_{3}^{\prime} & A_{1}
\end{array}\right)
$$

where $A_{i}=\operatorname{cir}\left(a_{3 i-2}, a_{3 i-1}, a_{3 i}\right)$ and $A_{i}^{\prime}=\operatorname{cir}\left(a_{3 i}, a_{3 i-2}, a_{3 i-1}\right)$.

Table 1: Extremal binary self-dual codes of length 20 from $C_{9}$.

\begin{tabular}{ccccc}
\hline$\left(\gamma_{1}, \alpha_{1}, \gamma_{2}, \alpha_{2}\right)$ & $\left(a_{1}, a_{2}, a_{3}\right)$ & $\left(a_{4}, a_{5}, a_{6}\right)$ & $\left(a_{7}, a_{8}, a_{9}\right)$ & $\mid$ Aut $(C) \mid$ \\
\hline$(1,0,0,1)$ & $(0,0,0)$ & $(0,0,1)$ & $(1,1,1)$ & $2^{10} \cdot 3^{4}$ \\
$(1,0,0,1)$ & $(0,1,1)$ & $(1,1,1)$ & $(1,1,1)$ & $2^{17} \cdot 3^{4} \cdot 5^{2} \cdot 7$ \\
\hline
\end{tabular}


Table 2: Self-dual codes over $\mathbb{F}_{2}+u \mathbb{F}_{2}$ of length 20 from $C_{9}$, whose binary images are extremal self-dual codes of length 40

\begin{tabular}{cccccc}
\hline$\left(\gamma, \alpha_{1}, \gamma_{2}, \alpha_{2}\right)$ & $\left(a_{1}, a_{2}, a_{3}\right)$ & $\left(a_{4}, a_{5}, a_{6}\right)$ & $\left(a_{7}, a_{8}, a_{9}\right)$ & $|A u t(C)|$ & Type \\
\hline$(1, u, u, 1)$ & $(u, 0,0)$ & $(u, u, 1)$ & $(1+u, 1,1+u)$ & $2^{2} \cdot 3^{2}$ & Type I \\
$(1, u, 0,1)$ & $(u, u, 0)$ & $(u, u, 1)$ & $(1+u, 1+u, 1)$ & $2^{3} \cdot 3^{2} \cdot 5 \cdot 19$ & Type II \\
$(1, u, 0,1)$ & $(u, u, 0)$ & $(0,0,1)$ & $(1+u, 1+u, 1)$ & $2^{2} \cdot 3^{2}$ & Type II \\
$(1, u, u, 1)$ & $(u, 1,1)$ & $(1,1,1+u)$ & $(1+u, 1,1)$ & $2^{11} \cdot 3^{2}$ & Type I \\
$(1, u, 0,1)$ & $(0,1,1)$ & $(1,1,1+u)$ & $(1+u, 1,1)$ & $2^{11} \cdot 3^{2}$ & Type II \\
\hline
\end{tabular}

- Let $G=C_{3} \times C_{3} \cong\left\langle x, y \mid x^{3}=y^{3}=1, x y=y x\right\rangle$. If $\alpha=\sum_{i=0}^{2} \sum_{j=0}^{2} a_{i+3 j+1} x^{i} y^{j} \in$ $R\left(C_{3} \times C_{3}\right)$, then

$$
\sigma(\alpha)=C I R\left(A_{1}, A_{2}, A_{3}\right)
$$

where where $A_{i}=\operatorname{cir}\left(a_{3 i-2}, a_{3 i-1}, a_{3 i}\right)$.

Table 3: Extremal binary self-dual codes of length 20 from $C_{3} \times C_{3}$.

\begin{tabular}{ccccc}
\hline$\left(\gamma_{1}, \alpha_{1}, \gamma_{2}, \alpha_{2}\right)$ & $\left(a_{1}, a_{2}, a_{3}\right)$ & $\left(a_{4}, a_{5}, a_{6}\right)$ & $\left(a_{7}, a_{8}, a_{9}\right)$ & $\mid$ Aut $(C) \mid$ \\
\hline$(1,0,0,1)$ & $(0,0,0)$ & $(0,0,1)$ & $(1,1,1)$ & $2^{10} \cdot 3^{4}$ \\
$(1,0,0,1)$ & $(0,1,1)$ & $(1,1,1)$ & $(1,1,1)$ & $2^{17} \cdot 3^{4} \cdot 5^{2} \cdot 7$ \\
\hline
\end{tabular}

Table 4: Self-dual codes over $\mathbb{F}_{2}+u \mathbb{F}_{2}$ of length 20 from $C_{3} \times C_{3}$, whose binary images are extremal self-dual codes of length 40

\begin{tabular}{cccccc}
\hline$\left(\gamma_{1}, \alpha_{1}, \gamma_{2}, \alpha_{2}\right)$ & $\left(a_{1}, a_{2}, a_{3}\right)$ & $\left(a_{4}, a_{5}, a_{6}\right)$ & $\left(a_{7}, a_{8}, a_{9}\right)$ & $\mid$ Aut $(C) \mid$ & Type \\
\hline$(1, u, u, 1)$ & $(u, 0,0)$ & $(u, u, 1)$ & $(1+u, 1,1+u)$ & $2^{3} \cdot 3^{2}$ & Type I \\
$(1, u, 0,1)$ & $(u, u, 0)$ & $(u, u, 1)$ & $(1+u, 1+u, 1)$ & $2^{4} \cdot 3^{4}$ & Type II \\
$(1, u, 0,1)$ & $(u, u, 0)$ & $(0,0,1)$ & $(1+u, 1+u, 1)$ & $2^{3} \cdot 3^{2}$ & Type II \\
$(1, u, u, 1)$ & $(u, 1,1)$ & $(1,1+u, 1+u)$ & $(1,1+u, 1+u)$ & $2^{15} \cdot 3^{3} \cdot 5$ & Type I \\
$(1, u, 0,1)$ & $(0,1,1)$ & $(1,1+u, 1+u)$ & $(1,1+u, 1+u)$ & $2^{15} \cdot 3^{3} \cdot 5$ & Type II \\
\hline
\end{tabular}

\subsection{Constructions from Groups of Order 15}

In this subsection and in some other subsections/sections extremal self-dual codes of length 64 will be constructed. We would like to recall that the possible weight enumerators for a 
self-dual Type I [64, 32, 12]-code is given in [4] as:

$$
\begin{aligned}
& W_{64,1}=1+(1312+16 \beta) y^{12}+(22016-64 \beta) y^{14}+\cdots, 14 \leq \beta \leq 284 \\
& W_{64,2}=1+(1312+16 \beta) y^{12}+(23040-64 \beta) y^{14}+\cdots, 0 \leq \beta \leq 277
\end{aligned}
$$

With the most updated information, the existence of codes is known for $\beta=14,18,22,25$, $29,32,35,36,39,44,46,53,59,60,64$ and 74 in $W_{64,1}$ and for $\beta=0,1,2,4,5,6,8,9,10$, $12,13,14,16, \ldots, 25,28,19,30,32,33,34,36,37,38,40,41,42,44,45,48,50,51,52,56$, $58,64,72,80,88,96,104,108,112,114,118,120$ and 184 in $W_{64,2}$.

- Let $G=C_{15} \cong\left\langle x \mid x^{15}=1\right\rangle$. If $\alpha=\sum_{i=0}^{4} \sum_{j=0}^{2} a_{i+5 j+1} x^{3 i+j} \in R C_{15}$, then

$$
\sigma(\alpha)=\left(\begin{array}{ccc}
A_{1} & A_{2} & A_{3} \\
A_{3}^{\prime} & A_{1} & A_{2} \\
A_{2}^{\prime} & A_{3}^{\prime} & A_{1}
\end{array}\right)
$$

where $A_{i}=\operatorname{cir}\left(a_{5 i-4}, a_{5 i-3}, a_{5 i-2}, a_{5 i-1}, a_{5 i}\right)$ and $A_{i}^{\prime}=\operatorname{cir}\left(a_{5 i}, a_{5 i-4}, a_{5 i-3}, a_{5 i-2}, a_{5 i-1}\right)$.

Table 5: Self-dual codes over $\mathbb{F}_{2}+u \mathbb{F}_{2}$ of length 32 from $C_{15}$ where $|A u t(C)|=2^{2} \cdot 3 \cdot 5$ and $\left(\gamma_{1}, \alpha_{1}, \gamma_{2}, \alpha_{2}\right)=(1, u, u, 1)$, whose binary images are Type II extremal self-dual of length 64

\begin{tabular}{ccc}
\hline$\left(a_{1}, \ldots, a_{5}\right)$ & $\left(a_{6}, \ldots, a_{10}\right)$ & $\left(a_{11}, \ldots, a_{15}\right)$ \\
\hline$(u, u, 0, u, 1)$ & $(u, u, u+1, u, u+1)$ & $(0,1, u, 1,1)$ \\
\hline$(u, u, 0, u, 1)$ & $(u, 0,1, u, u+1)$ & $(u, 1, u, 1, u+1)$ \\
\hline$(u, u, 0, u, 1)$ & $(0,0,1,0, u+1)$ & $(u, u+1, u, u+1, u+1)$ \\
\hline
\end{tabular}

- Let $G=C_{3} \times C_{5} \cong\left\langle x, y \mid x^{5}=y^{3}=1, x y=y x\right\rangle$. If $\alpha=\sum_{i=0}^{2} \sum_{j=0}^{4} a_{i+3 j+1} x^{i} y^{j} \in$ $R\left(C_{3} \times C_{5}\right)$, then

$$
\sigma(\alpha)=C I R\left(A_{1}, A_{2}, A_{3}, A_{4}, A_{5}\right)
$$

where $A_{i}=\operatorname{cir}\left(a_{3 i-2}, a_{3 i-1}, a_{3 i}\right)$.

Table 6: Type II extremal binary self-dual codes of length 32 from groups of order 15

\begin{tabular}{cccc}
\hline$G$ & $\left(\gamma_{1}, \alpha_{1}, \gamma_{2}, \alpha_{2}\right)$ & $\left(a_{1}, \ldots, a_{15}\right)$ & $\mid$ Aut $(C) \mid$ \\
\hline$C_{15}$ & $(1,0,0,1)$ & $(0,0,0,0,1,0,0,1,0,1,0,1,0,1,1)$ & $2^{5} \cdot 3 \cdot 5 \cdot 31$ \\
\hline$C_{3} \times C_{3}$ & $(1,0,0,1)$ & $(0,0,1,0,0,1,0,0,1,0,1,0,1,1,0)$ & $2^{5} \cdot 3 \cdot 5 \cdot 31$ \\
\hline
\end{tabular}


Table 7: Self-dual codes over $\mathbb{F}_{2}+u \mathbb{F}_{2}$ of length 32 where $|A u t(C)|=2^{2} \cdot 3 \cdot 5$ and $\left(\gamma_{1}, \alpha_{1}, \gamma_{2}, \alpha_{2}\right)=(1, u, 0,1)$, whose binary images are Type I

\begin{tabular}{cccc}
\hline $\mathcal{C}_{i}$ & $G$ & $\left(a_{1}, a_{2}, \ldots, a_{15}\right)$ & $\beta$ in $W_{64,1}$ \\
\hline $\mathcal{C}_{1}$ & $C_{15}$ & $(u, u, 0,0,1, u, 0,1, u, 1,0,1,0,1,1)$ & 14 \\
$\mathcal{C}_{2}$ & $C_{15}$ & $(u, u, 0, u, 1,0, u, 1+u, 0,1, u, 1, u, 1,1+u)$ & 44 \\
$\mathcal{C}_{3}$ & $C_{3} \times C_{5}$ & $(u, 0,1,0, u, 1+u, u, u, 1, u, 1,0,1+u, 1, u)$ & 14 \\
$\mathcal{C}_{4}$ & $C_{3} \times C_{5}$ & $(0,0,1, u, 0,1, u, u, 1+u, u, 1, u, 1,1+u, u)$ & 44 \\
\hline
\end{tabular}

\subsection{Constructions from Groups of Order 21}

- Let $G=C_{21} \cong\left\langle x \mid x^{21}=1\right\rangle$. If $\alpha=\sum_{i=0}^{6} \sum_{j=0}^{2} a_{i+7 j+1} x^{3 i+j} \in R C_{21}$, then

$$
\sigma(\alpha)=\left(\begin{array}{ccc}
A_{1} & A_{2} & A_{3} \\
A_{3}^{\prime} & A_{1} & A_{2} \\
A_{2}^{\prime} & A_{3}^{\prime} & A_{1}
\end{array}\right)
$$

where $A_{i}=\operatorname{cir}\left(a_{7 i-6}, a_{7 i-5}, \ldots, a_{7 i}\right)$ and $A_{i}^{\prime}=\operatorname{cir}\left(a_{7 i}, a_{7 i-6}, \ldots, a_{7 i-1}\right)$.

Table 8: Extremal binary self-dual codes of length 44 from $C_{21}$.

\begin{tabular}{ccccc}
\hline$\left.\gamma_{1}, \alpha_{1}, \gamma_{2}, \alpha_{2}\right)$ & $\left(a_{1}, \ldots, a_{7}\right)$ & $\left(a_{8}, \ldots, a_{14}\right)$ & $\left(a_{15}, \ldots, a_{21}\right)$ & $\mid A u(t(C) \mid$ \\
\hline$(1,0,0,1)$ & $(0,0,0,0,0,0,1)$ & $(0,0,1,1,1,1,1)$ & $(0,0,1,1,0,0,0)$ & $2 \cdot 3 \cdot 7$ \\
$(1,0,0,1)$ & $(0,0,0,0,0,1,1)$ & $(0,1,0,1,1,0,0)$ & $(1,0,0,1,1,0,0)$ & $2 \cdot 3 \cdot 7$ \\
$(1,0,0,1)$ & $(0,0,0,0,0,1,1)$ & $(1,1,0,1,1,1,0)$ & $(0,1,1,1,1,0,1)$ & $2 \cdot 3 \cdot 7$ \\
$(1,0,0,1)$ & $(0,0,0,0,1,1,1)$ & $(1,0,0,1,0,1,1)$ & $(1,1,1,0,1,0,1)$ & $2 \cdot 3 \cdot 7$ \\
$(1,0,0,1)$ & $(0,0,0,1,0,1,1)$ & $(0,1,0,1,0,1,1)$ & $(1,1,0,1,1,0,1)$ & $2 \cdot 3 \cdot 7$ \\
\hline
\end{tabular}


Table 9: Self-dual codes over $\mathbb{F}_{2}+u \mathbb{F}_{2}$ of length 44 from $C_{21}$ where $\left(\gamma_{1}, \alpha_{1}, \gamma_{2}\right)=(1, u, 0,1)$ and $\mid$ Aut $(C) \mid=2^{2} \cdot 3 \cdot 7$, whose binary images are Type II extremal self-dual of length 88 .

\begin{tabular}{ccc}
\hline$\left(a_{1}, \ldots, a_{7}\right)$ & $\left(a_{8}, \ldots, a_{14}\right)$ & $\left(a_{15}, \ldots, a_{21}\right)$ \\
\hline$(u, u, 0, u, u, u, 1)$ & $(u, u, 1,1,1,1, u+1)$ & $(u, u, 1, u+1, u, 0,0)$ \\
\hline$(u, u, 0, u, u, u, 1)$ & $(u, u, u+1,1,1, u+1, u+1)$ & $(0, u, 1, u+1,0,0,0)$ \\
\hline$(u, u, u, u, 1,1, u+1)$ & $(1,0, u, 1, u, u+1, u+1)$ & $(1, u+1, u+1,0, u+1,0,1)$ \\
\hline$(u, u, u, u, 1,1, u+1)$ & $(1,0,0, u+1,0, u+1,1)$ & $(1,1, u+1, u, u+1, u, u+1)$ \\
\hline$(u, u, u, u, u, 1, u+1)$ & $(1,1,0,1,1, u+1,0)$ & $(0, u+1, u+1,1, u+1, u, u+1)$ \\
\hline$(u, u, u, u, 0,1,1)$ & $(1,1, u, 1,1, u+1,0)$ & $(0, u+1, u+1, u+1, u+1, u, u+1)$ \\
\hline$(u, u, u, u, u, 1, u+1)$ & $(0,1,0, u+1,1,0, u)$ & $(1,0, u, u+1, u+1,0, u)$ \\
\hline$(u, u, u, u, u, 1, u+1)$ & $(0, u+1,0, u+1,1, u, 0)$ & $(1, u, 0, u+1,1, u, 0)$ \\
\hline$(u, u, u, 1, u, 1,1)$ & $(u, u+1, u, 1,0, u+1,1)$ & $(u+1, u+1, u, 1,1, u, 1)$ \\
\hline$(u, u, u, 1, u, 1, u+1)$ & $(u, 1,0, u+1, u, 1, u+1)$ & $(u+1,1,0,1, u+1,0, u+1)$ \\
\hline
\end{tabular}

- Let $G=F_{21} \cong\left\langle x, y \mid x^{3}=y^{7}=1, y x=x y^{2}\right\rangle$. If $\alpha=\sum_{i=0}^{6} \sum_{j=0}^{2} a_{i+y j+1} y^{i} x^{j} \in R F_{21}$, then

$$
\sigma(\alpha)=\left(\begin{array}{ccc}
A_{1} & A_{2} & A_{3} \\
A_{3}^{\prime} & A_{1}^{\prime} & A_{2}^{\prime} \\
A_{2}^{\prime \prime} & A_{3}^{\prime \prime} & A_{1}^{\prime \prime}
\end{array}\right)
$$

where $A_{i}=\operatorname{cir}\left(a_{7 i-6}, a_{7 i-5}, \ldots, a_{7 i}\right), A_{i}^{\prime}=\operatorname{cir}_{2}\left(a_{7 i-6}, a_{7 i-5}, \ldots, a_{7 i}\right)$ and $A_{i}^{\prime}=\operatorname{cir}_{4}\left(a_{7 i-6}, a_{7 i-5}, \ldots, a_{7 i}\right)$

Table 10: Extremal binary self-dual code of length 44 from $F_{21}$.

\begin{tabular}{ccccc}
\hline$\left(\gamma_{1}, \alpha_{1}, \gamma_{2}, \alpha_{2}\right)$ & $\left(a_{1}, \ldots, a_{7}\right)$ & $\left(a_{8}, \ldots, a_{14}\right)$ & $\left(a_{15}, \ldots, a_{21}\right)$ & $\mid$ Aut $(C) \mid$ \\
\hline$(1,0,0,1)$ & $(0,0,0,0,0,1,1)$ & $(0,1,0,1,1,1,1)$ & $(1,1,0,1,1,1,0)$ & $2^{5} \cdot 3 \cdot 7$ \\
\hline
\end{tabular}


Table 11: Self-dual codes over $\mathbb{F}_{2}+u \mathbb{F}_{2}$ of length 44 from $F_{21}$ where $\left(\gamma_{1}, \alpha_{1}, \gamma_{2}\right)=(1, u, 0,1)$, whose binary images are Type II extremal self-dual of length 88 .

\begin{tabular}{cccc}
\hline$\left(a_{1}, \ldots, a_{7}\right)$ & $\left(a_{8}, \ldots, a_{14}\right)$ & $\left(a_{15}, \ldots, a_{21}\right)$ & $\mid$ Aut $(C) \mid$ \\
\hline$(u, u, 0, u, 0,1, u+1)$ & $(u, u+1, u, u+1,1, u+1,1)$ & $(1,1, u, 1, u+1, u+1,0)$ & $2 \cdot 3 \cdot 7$ \\
\hline$(u, u, 0, u, 0,1, u+1)$ & $(u, u+1, u, u+1,1, u+1,1)$ & $(u+1, u+1,0, u+1, u+1,1, u)$ & $2 \cdot 3 \cdot 7$ \\
\hline$(u, u, 0,0,0,1,1)$ & $(u, 1, u, u+1, u+1,1,1)$ & $(1, u+1, u, u+1,1,1, u)$ & $2^{2} \cdot 3 \cdot 7$ \\
\hline$(u, u, 0,0,0,1,1)$ & $(u, 1,0,1,1, u+1,1)$ & $(u+1, u+1,0, u+1,1,1, u)$ & $2 \cdot 3 \cdot 7$ \\
\hline$(u, u, 0,0,0,1,1)$ & $(u, u+1,0,1,1, u+1,1)$ & $(1,1, u, 1, u+1, u+1,0)$ & $2^{2} \cdot 3 \cdot 7$ \\
\hline$(u, 0,0, u, 0,1,1)$ & $(u, u+1, u, 1,1, u+1, u+1)$ & $(1, u+1,0,1, u+1, u+1,0)$ & $2 \cdot 3 \cdot 7$ \\
\hline$(0, u, 0,0,0,1, u+1)$ & $(u, u+1, u, 1, u+1, u+1,1)$ & $(1, u+1, u, 1, u+1,1,0)$ & $2 \cdot 3 \cdot 7$ \\
\hline$(0,0,0,0,0,1,1)$ & $(u, u+1,0,1,1,1, u+1)$ & $(u+1,1, u, 1, u+1,1,0)$ & $2^{2} \cdot 3 \cdot 7$ \\
\hline
\end{tabular}

\subsection{Constructions from Groups of Order 25}

- Let $G=C_{25} \cong\left\langle x \mid x^{25}=1\right\rangle$. If $\alpha=\sum_{i=0}^{4} \sum_{j=0}^{4} a_{i+5 j+1} x^{5 i+j} \in R C_{25}$, then

$$
\sigma(\alpha)=\left(\begin{array}{ccccc}
A_{1} & A_{2} & A_{3} & A_{4} & A_{5} \\
A_{5}^{\prime} & A_{1} & A_{2} & A_{3} & A_{4} \\
A_{4}^{\prime} & A_{5}^{\prime} & A_{1} & A_{2} & A_{3} \\
A_{3}^{\prime} & A_{4}^{\prime} & A_{5}^{\prime} & A_{1} & A_{2} \\
A_{2}^{\prime} & A_{3}^{\prime} & A_{4}^{\prime} & A_{5}^{\prime} & A_{1}
\end{array}\right)
$$

where $A_{i}=\operatorname{cir}\left(a_{5 i-4}, a_{5 i-3}, a_{5 i-2}, a_{5 i-1}, a_{5 i}\right)$ and $A_{i}^{\prime}=\operatorname{cir}\left(a_{5 i}, a_{5 i-4}, a_{5 i-3}, a_{5 i-2}, a_{5 i-1}\right)$.

- Let $G=C_{5} \times C_{5} \cong\left\langle x, y \mid x^{5}=y^{5}=1, x y=y x\right\rangle$. If $\alpha=\sum_{i=0}^{4} \sum_{j=0}^{4} a_{i+5 j+1} x^{j} y^{i} \in$ $R\left(C_{5} \times C_{5}\right)$, then

$$
\sigma(\alpha)=C I R\left(A_{1}, A_{2}, A_{3}, A_{4}, A_{5}\right)
$$

where $A_{i}=\operatorname{cir}\left(a_{5 i-4}, a_{5 i-3}, a_{5 i-2}, a_{5 i-1}, a_{5 i}\right)$.

Table 12: Extremal binary self-dual codes of length 52 where $\left(\gamma_{1}, \alpha_{1}, \gamma_{2}, \alpha_{2}\right)=(1,0,0,1)$.

\begin{tabular}{cccc}
\hline$G$ & $\left(a_{1}, \ldots, a_{25}\right)$ & $|A u t(C)|$ & Type \\
\hline$C_{25}$ & $(0,0,0,0,0,0,0,0,0,1,0,0,0,0,1,1,1,1,0,0,1,0,1,1,0)$ & $2 \cdot 5^{2}$ & $W_{52,1}$ \\
$C_{25}$ & $(0,0,0,0,1,0,0,1,1,1,1,0,1,1,1,1,1,1,1,1,1,0,1,0,1)$ & $2 \cdot 5^{2}$ & $W_{52,1}$ \\
$C_{5} \times C_{5}$ & $(0,0,0,0,1,0,0,0,0,1,0,0,0,1,1,0,1,1,1,0,1,0,1,1,1)$ & $2 \cdot 3 \cdot 5^{2}$ & $W_{52,1}$ \\
\hline
\end{tabular}




\subsection{Constructions from Groups of Order 27}

- Let $G=C_{27} \cong\left\langle x \mid x^{27}=1\right\rangle$. If $\alpha=\sum_{i=0}^{2} \sum_{j=0}^{8} a_{i+3 j+1} x^{9 i+j} \in R C_{27}$, then

$$
\sigma(\alpha)=\left(\begin{array}{lllllllll}
A_{1} & A_{2} & A_{3} & A_{4} & A_{5} & A_{6} & A_{7} & A_{8} & A_{9} \\
A_{9}^{\prime} & A_{1} & A_{2} & A_{3} & A_{4} & A_{5} & A_{6} & A_{7} & A_{8} \\
A_{8}^{\prime} & A_{9}^{\prime} & A_{1} & A_{2} & A_{3} & A_{4} & A_{5} & A_{6} & A_{7} \\
A_{7}^{\prime} & A_{8}^{\prime} & A_{9}^{\prime} & A_{1} & A_{2} & A_{3} & A_{4} & A_{5} & A_{6} \\
A_{6}^{\prime} & A_{7}^{\prime} & A_{8}^{\prime} & A_{9}^{\prime} & A_{1} & A_{2} & A_{3} & A_{4} & A_{5} \\
A_{5}^{\prime} & A_{6}^{\prime} & A_{7}^{\prime} & A_{8}^{\prime} & A_{9}^{\prime} & A_{1} & A_{2} & A_{3} & A_{4} \\
A_{4}^{\prime} & A_{5}^{\prime} & A_{6}^{\prime} & A_{7}^{\prime} & A_{8}^{\prime} & A_{9}^{\prime} & A_{1} & A_{2} & A_{3} \\
A_{3}^{\prime} & A_{4}^{\prime} & A_{5}^{\prime} & A_{6}^{\prime} & A_{7}^{\prime} & A_{8}^{\prime} & A_{9}^{\prime} & A_{1} & A_{2} \\
A_{2}^{\prime} & A_{3}^{\prime} & A_{4}^{\prime} & A_{5}^{\prime} & A_{6}^{\prime} & A_{7}^{\prime} & A_{8}^{\prime} & A_{9}^{\prime} & A_{1}
\end{array}\right)
$$

where $A_{i}=\operatorname{cir}\left(a_{3 i-2}, a_{3 i-1}, a_{3 i}\right)$ and $A_{i}^{\prime}=\operatorname{cir}\left(a_{3 i}, a_{3 i-2}, a_{3 i-1}\right)$.

- Let $G=\left(C_{3} \times C_{3}\right) \rtimes C_{3}=\left\langle x, y, z \mid x^{3}=y^{3}=z^{3}=1, y z=z y x, x y=y x, x z=z x\right\rangle$. If $\alpha=\sum_{i=0}^{2} \sum_{j=0}^{2} \sum_{k=0}^{2} a_{1+3 i+9 j+k} x^{i} y^{j} z^{k} \in R\left(\left(C_{3} \times C_{3}\right) \rtimes C_{3}\right)$, then

$$
\sigma(\alpha)=\left(\begin{array}{ccc}
M_{1} & M_{2} & M_{3} \\
M_{4} & M_{5} & M_{6} \\
M_{7} & M_{8} & M_{9}
\end{array}\right)
$$

where $M_{1}=\operatorname{CIR}\left(A_{1}, A_{2}, A_{3}\right), M_{2}=\operatorname{CIR}\left(A_{4}, A_{5}, A_{6}\right), M_{3}=C I R\left(A_{7}, A_{8}, A_{9}\right)$, $M_{4}=\operatorname{CIR}\left(A_{7}, A_{8}^{(1)}, A_{9}^{(2)}\right), M_{5}=\operatorname{CIR}\left(A_{1}, A_{2}^{(1)}, A_{3}^{(2)}\right), M_{6}=C I R\left(A_{4}, A_{5}^{(1)}, A_{6}^{(2)}\right)$, $M_{7}=\operatorname{CIR}\left(A_{4}, A_{5}^{(2)}, A_{6}^{(1)}\right), M_{8}=\operatorname{CIR}\left(A_{7}, A_{8}^{(2)}, A_{9}^{(1)}\right)$ and $M_{9}=C I R\left(A_{1}, A_{2}^{(2)}, A_{3}^{(1)}\right)$, $A_{i}=\operatorname{cir}\left(a_{3 i-2}, a_{3 i-1}, a_{3 i}\right), A_{i}^{(1)}=\operatorname{cir}\left(a_{3 i}, a_{3 i-2}, a_{3 i-1}\right)$ and $A_{i}^{(2)}=\operatorname{cir}\left(a_{3 i-1}, a_{3 i}, a_{3 i-2}\right)$.

- Let $G=C_{9} \rtimes C_{3} \cong\left\langle x, y \mid x^{3}=y^{9}=1, y x=x y^{4}\right\rangle$. If $\alpha=\sum_{i=0}^{8} \sum_{j=0}^{2} a_{i+y j+1} y^{i} x^{j} \in$ $R\left(C_{9} \rtimes C_{3}\right)$, then

$$
\sigma(\alpha)=\left(\begin{array}{ccc}
A_{1} & A_{2} & A_{3} \\
A_{3}^{\prime} & A_{1}^{\prime} & A_{3}^{\prime} \\
A_{2}^{\prime \prime} & A_{3}^{\prime \prime} & A_{1}^{\prime \prime}
\end{array}\right)
$$

where $A_{i}=\operatorname{cir}\left(a_{9 n-8}, \ldots, a_{9 i}\right), A_{i}^{\prime}=\operatorname{cir}_{2}\left(a_{9 n-8}, \ldots, a_{9 i}\right)$ and $A_{i}^{\prime \prime}=5-\operatorname{cir}\left(a_{9 n-8}, \ldots, a_{9 i}\right)$.

- Let $G=C_{3}^{3}=\left\langle x, y, z \mid x^{3}=y^{3}=z^{3}=1, y z=z y, x y=y x, x z=z x\right\rangle$. If $\alpha=$ $\sum_{i=0}^{2} \sum_{j=0}^{2} \sum_{k=0}^{2} a_{1+i+3 j+9 k} x^{i} y^{j} z^{k} \in R C_{3}^{3}$, then

$$
\sigma(\alpha)=C I R\left(M_{1}, M_{2}, M_{3}\right)
$$

where $M_{1}=\operatorname{CIR}\left(A_{1}, A_{2}, A_{3}\right), M_{2}=\operatorname{CIR}\left(A_{4}, A_{5}, A_{6}\right)$ and $M_{3}=\operatorname{CIR}\left(A_{7}, A_{8}, A_{9}\right)$, $A_{i}=\operatorname{cir}\left(a_{3 i-2}, a_{3 i-1}, a_{3 i}\right)$. 
Table 13: Type II self-dual $[56,28,12]$ codes where $\left(\gamma_{1}, \alpha_{1}, \gamma_{2}, \alpha_{2}\right)=(1,0,0,1)$.

\begin{tabular}{ccc}
\hline$G$ & $\left(a_{1}, \ldots, a_{27}\right)$ & $\mid$ Aut $(C) \mid$ \\
\hline$C_{27}$ & $(0,0,0,0,0,1,1,0,0,0,1,1,0,1,0,0,1,1,1,1,0,0,0,1,0,0,0)$ & $2 \cdot 3^{3}$ \\
$C_{27}$ & $(0,0,0,0,0,1,0,0,1,1,0,1,1,0,1,0,0,1,0,1,1,1,1,0,1,1,1)$ & $2 \cdot 3^{3}$ \\
$\left(C_{3} \times C_{3}\right) \rtimes C_{3}$ & $(0,0,1,0,0,1,1,0,0,0,0,1,0,1,1,1,1,0,1,0,1,0,1,1,0,1,1)$ & $2 \cdot 3^{3}$ \\
$C_{9} \rtimes C_{3}$ & $(0,0,0,0,0,0,0,1,1,0,0,0,0,1,0,0,1,1,0,1,0,1,1,1,1,0,0)$ & $2 \cdot 3^{3}$ \\
$C_{9} \rtimes C_{3}$ & $(0,0,0,0,0,0,0,1,1,0,0,0,0,1,0,0,1,1,1,1,0,0,1,1,1,0,0)$ & $2^{3} \cdot 3^{3} \cdot 7$ \\
$C_{9} \rtimes C_{3}$ & $(0,0,0,0,0,0,0,1,1,0,0,1,1,0,1,1,1,0,1,0,1,1,1,0,1,1,1)$ & $3^{3}$ \\
$C_{9} \rtimes C_{3}$ & $(0,0,0,0,0,1,0,1,1,0,1,1,1,0,0,1,0,1,1,0,1,1,0,0,1,1,1)$ & $2^{3} \cdot 3^{4} \cdot 7 \cdot 13$ \\
$C_{3}^{3}$ & $(0,0,0,0,0,1,1,0,1,0,0,1,0,1,1,1,1,0,0,1,1,1,1,1,0,1,0)$ & $2^{3} \cdot 3^{4} \cdot 7 \cdot 13$ \\
\hline
\end{tabular}

\subsection{Constructions from Groups of Order 33}

Let $G=C_{33} \cong\left\langle x \mid x^{33}=1\right\rangle$. If $\alpha=\sum_{i=0}^{10} \sum_{j=0}^{2} a_{i+11 j+1} x^{3 i+j} \in R C_{21}$, then

$$
\sigma(\alpha)=\left(\begin{array}{ccc}
A_{1} & A_{2} & A_{3} \\
A_{3}^{\prime} & A_{1} & A_{2} \\
A_{2}^{\prime} & A_{3}^{\prime} & A_{1}
\end{array}\right)
$$

where $A_{i}=\operatorname{cir}\left(a_{9 n-8}, \ldots, a_{9 i}\right)$ and $A_{i}^{\prime}=\operatorname{cir}\left(a_{9 i}, a_{9 n-8}, \ldots, a_{9 i-1}\right)$.

Table 14: Extremal binary self-dual codes of length 68 from $C_{33}$ where $\left(\gamma_{1}, \alpha_{1}, \gamma_{2}, \alpha_{2}\right)=$ $(1,0,0,1)$.

\begin{tabular}{cccc}
\hline $\mathcal{D}_{i}$ & $\left(a_{1}, \ldots, a_{33}\right)$ & $|A u t(C)|$ & $W_{68,1}$ \\
\hline $\mathcal{D}_{1}$ & $(0,0,0,0,0,0,0,0,0,0,1,0,0,0,0,0,1,0,1,1,0,1,0,1,1,0,1,1,0,0,1,1,1)$ & $2 \cdot 3 \cdot 11$ & $\beta=137$ \\
$\mathcal{D}_{2}$ & $(0,0,0,0,0,0,0,0,0,0,1,0,0,0,0,0,1,1,1,1,1,1,1,0,1,1,0,0,1,1,0,0,0)$ & $2 \cdot 3 \cdot 11$ & $\beta=170$ \\
$\mathcal{D}_{3}$ & $(0,0,0,0,0,0,0,0,0,0,1,0,0,0,0,1,0,0,1,0,1,1,0,1,1,1,0,0,1,1,1,0,1)$ & $2 \cdot 3 \cdot 11$ & $\beta=170$ \\
$\mathcal{D}_{4}$ & $(0,0,0,0,0,0,0,0,0,0,1,0,0,0,1,1,1,0,0,0,1,1,1,0,0,1,0,1,1,1,0,1,0)$ & $2 \cdot 3 \cdot 11$ & $\beta=236$ \\
$\mathcal{D}_{5}$ & $(0,0,0,0,0,0,0,0,0,0,1,0,1,1,1,0,1,0,0,1,0,0,1,1,0,1,1,1,1,1,1,1,1)$ & $2 \cdot 3 \cdot 5 \cdot 11$ & $\beta=104$ \\
$\mathcal{D}_{6}$ & $(0,0,0,0,0,0,0,0,1,1,1,0,0,0,0,0,0,1,0,1,1,0,0,0,0,1,1,1,0,1,1,1,0)$ & $2 \cdot 3 \cdot 11$ & $\beta=203$ \\
$\mathcal{D}_{7}$ & $(0,0,0,0,0,0,0,1,0,1,1,0,1,1,0,0,0,0,0,1,0,1,1,1,0,0,1,0,0,1,0,1,0)$ & $2 \cdot 3 \cdot 11$ & $\beta=335$ \\
$\mathcal{D}_{8}$ & $(0,0,0,0,0,0,0,1,0,1,1,0,0,1,0,1,0,0,0,0,0,1,0,0,1,0,0,1,1,1,1,1,0)$ & $2 \cdot 3 \cdot 11$ & $\beta=269$ \\
\hline
\end{tabular}




\subsection{Constructions from Groups of Order 35}

Let $G=C_{35} \cong\left\langle x \mid x^{35}=1\right\rangle$. If $\alpha=\sum_{i=0}^{4} \sum_{j=0}^{6} a_{i+5 j+1} x^{7 i+j} \in R C_{35}$, then

$$
\sigma(\alpha)=\left(\begin{array}{lllllll}
A_{1} & A_{2} & A_{3} & A_{4} & A_{5} & A_{6} & A_{7} \\
A_{7}^{\prime} & A_{1} & A_{2} & A_{3} & A_{4} & A_{5} & A_{6} \\
A_{6}^{\prime} & A_{7}^{\prime} & A_{1} & A_{2} & A_{3} & A_{4} & A_{5} \\
A_{5}^{\prime} & A_{6}^{\prime} & A_{7}^{\prime} & A_{1} & A_{2} & A_{3} & A_{4} \\
A_{4}^{\prime} & A_{5}^{\prime} & A_{6}^{\prime} & A_{7}^{\prime} & A_{1} & A_{2} & A_{3} \\
A_{3}^{\prime} & A_{4}^{\prime} & A_{5}^{\prime} & A_{6}^{\prime} & A_{7}^{\prime} & A_{1} & A_{2} \\
A_{2}^{\prime} & A_{3}^{\prime} & A_{4}^{\prime} & A_{5}^{\prime} & A_{6}^{\prime} & A_{7}^{\prime} & A_{1}
\end{array}\right)
$$

where $A_{i}=\operatorname{cir}\left(a_{5 i-4}, a_{5 i-3}, a_{5 i-2}, a_{5 i-1}, a_{5 i}\right)$ and $A_{i}^{\prime}=\operatorname{cir}\left(a_{5 i}, a_{5 i-4}, a_{5 i-3}, a_{5 i-2}, a_{5 i-1}\right)$.

Using the construction above, we get a number of Type II self-dual codes of parameters $[72,36,12]$, which are best known as it is still not known whether extremal binary self-dual codes of length 72 exist. The possible weight enumerators for a Type II $[72,36,12]$ code are given in [8] as

$$
W_{72}=1+(4398+\alpha) y^{12}+(197073-12 \alpha) y^{16}+\cdots,
$$

where $\alpha$ is a parameter.

Table 15: Type II self-dual $[72,36,12]$-codes from $C_{35}$ where $\left(\gamma_{1}, \alpha_{1}, \gamma_{2}, \alpha_{2}\right)=(1,0,0,1)$.

\begin{tabular}{ccc}
\hline$\left(a_{1}, \ldots, a_{35}\right)$ & $\mid$ Aut $(C) \mid$ & $\alpha \in W_{72}$ \\
\hline$(0,0,0,0,1,0,0,1,1,1,0,0,0,1,0,0,1,1,1,0,1,0,0,1,1,0,0,1,1,0,0,1,0,0,0)$ & $2 \cdot 5 \cdot 7$ & -3936 \\
$(0,0,0,0,1,0,0,1,1,1,0,0,0,0,0,1,0,1,1,0,1,1,0,1,0,1,0,0,0,0,0,1,0,1,1)$ & $2 \cdot 5 \cdot 7$ & -3726 \\
$(0,0,0,0,1,0,0,1,1,1,0,0,0,0,1,0,0,0,0,1,0,0,0,0,1,1,0,1,1,1,1,0,0,1,1)$ & $2 \cdot 5 \cdot 7$ & -3516 \\
$(0,0,0,0,1,0,0,1,1,1,0,0,0,0,0,1,1,1,1,1,1,0,0,1,1,0,0,0,0,1,0,0,1,0,0)$ & $2 \cdot 5 \cdot 7$ & -3306 \\
$(0,0,0,0,1,0,0,1,1,1,0,0,0,0,1,0,0,0,0,1,0,0,0,0,1,0,1,0,0,0,0,1,1,0,0)$ & $2 \cdot 5 \cdot 7$ & -3096 \\
$(0,0,0,0,1,0,0,1,1,1,0,0,0,0,1,0,1,1,1,0,1,1,0,1,1,0,0,1,1,1,1,1,0,0,1)$ & $2 \cdot 5 \cdot 7$ & -2886 \\
$(0,0,0,0,1,0,0,1,1,1,0,0,1,0,0,0,0,1,0,0,0,1,0,1,1,1,1,1,1,1,0,1,1,1,1)$ & $2 \cdot 5 \cdot 7$ & -2676 \\
$(0,0,0,0,1,0,0,1,1,1,0,0,1,1,1,0,1,1,0,1,0,1,1,1,0,0,0,1,1,0,1,0,1,1,0)$ & $2 \cdot 5 \cdot 7$ & -2466 \\
$(0,0,0,0,1,0,0,1,1,1,0,1,0,0,0,1,0,0,1,1,0,0,1,0,0,0,1,0,0,1,1,1,0,1,0)$ & $2^{3} \cdot 3^{2} \cdot 5 \cdot 7 \cdot 71$ & -1416 \\
\hline
\end{tabular}

\section{$5 \quad$ New binary self-dual codes of length 68}

The possible weight enumerator of an extremal self-dual-code of length 68 is in one of the following forms by $[3,15]$ :

$$
\begin{aligned}
& W_{68,1}=1+(442+4 \beta) y^{12}+(10864-8 \beta) y^{14}+\cdots, 104 \leq \beta \leq 1358, \\
& W_{68,2}=1+(442+4 \beta) y^{12}+(14960-8 \beta-256 \gamma) y^{14}+\cdots
\end{aligned}
$$

where $0 \leq \gamma \leq 9$. Recently, new codes with previously unknown weight enumerators in $W_{68,1}$ were constructed in [23]. Together with these the existence of codes in $W_{68,1}$ are known for 
$\beta=104,105,112,115,117,119,120,122,123,125, \ldots, 232,234,235,236,238,241,245$, $247,252,255,257, \ldots, 269,273,280,287,291,294,301,302,308,313,315,322,324,328, \ldots$, 336, 338, 339, 345, 347, 350, 355, 379 and 401.

Recently, Yankov et. al. constructed the first examples of codes with a weight enumerator for $\gamma=7$ in $W_{68,2}$. Together with these the existence of the codes in $W_{68,2}$ is known for;

$$
\begin{aligned}
\gamma= & 0, \beta=0,7,11,14,17,21,22,28,33,35,42,44, \ldots, 158,161,165, \\
& 175,187,189,203,209,221,231,255,303 \text { or } \\
\beta \in & \{2 m \mid m=17,20,102,110,119,136,165 \text { or } 80 \leq m \leq 99\} \\
\gamma= & 1, \beta=49,57,59, \ldots, 160 \text { or } \\
\beta \in & \{2 m \mid m=25, \ldots, 29,81, \ldots, 90,92, \ldots, 96\} ; \\
\gamma= & 2, \beta=65,69,71,77,81,159,206,208 \text { or } \beta \in\{2 m \mid 30 \leq m \leq 68,70 \leq m \leq 100\} \text { or } \\
\beta \in & \{2 m+1 \mid 42 \leq m \leq 69,71 \leq m \leq 77\} \\
\gamma= & 3, \beta=101,103,105,107,115,117,119,121,123,125,127,129,131,133,135 \\
& 137,139,141,143,145,147,149,151,153,155,159,161,193 \text { or } \\
\beta \in & \{2 m \mid m=44,45,47, \ldots, 84,86, \ldots, 92,94,95,97,98,101,102\} \\
\gamma= & 4, \beta=129,141,145,157,161 \text { or } \\
\beta= & \{2 m \mid m=43,48,49,51,52,54,55,56,58,60, \ldots, 78,80,87,97,98\} \\
\gamma= & 6 \text { with } \beta \in\{2 m \mid m=69,77,78,79,81,88\} \\
\gamma= & 7 \text { with } \beta \in\{7 m \mid m=14, \ldots, 39,42\} .
\end{aligned}
$$

In this section, we construct new extremal binary self-dual codes of length 68 from codes in Section 4. We were able to obtain 29 new codes as neighbours and 12 new codes by using the building-up construction (Theorem 2.5). All the codes found have weight enumerators, the existence of which was not known in the literature. The results are tabulated in Table 16 and Table 17, respectively.

More precisely, we obtain the codes for $\beta=233,237,239,240,242,243,244,246,248$, 249, 250, 251, 253, 254, 256, 270, 271, 272, 274,275, 276, 277, 278, 279, 281, 282, 283, 284, 289 in $W_{68,1}$ and for $\gamma=1$ and $\beta=51,53,55, \gamma=2$ and $\beta=73,75,79,83 ; \gamma=3$ and $\beta=84,95,97,109,113$ in $W_{68,2}$. 
Table 16: New self-dual $[68,34,12]_{2}$-codes as neighbors of $\mathcal{D}_{7}$ in Table 14

\begin{tabular}{lcc}
\hline $\mathcal{C}_{68, i}$ & $x_{35} \ldots x_{68}$ & $\beta$ in $W_{68,1}$ \\
\hline $\mathcal{C}_{68,1}$ & $(1001100001110001110011110010011011)$ & 233 \\
$\mathcal{C}_{68,2}$ & $(1110111101001001000010001111111000)$ & 237 \\
$\mathcal{C}_{68,3}$ & $(1111010100101011001001011100001101)$ & 239 \\
$\mathcal{C}_{68,4}$ & $(0110010111110000100000110101111000)$ & 240 \\
$\mathcal{C}_{68,5}$ & $(0010000000110110001111010100111101)$ & 242 \\
$\mathcal{C}_{68,6}$ & $(110100111011000000011111111111011)$ & 243 \\
$\mathcal{C}_{68,7}$ & $(1110111000000101101000110101101110)$ & 244 \\
$\mathcal{C}_{68,8}$ & $(1010010101011111111011111010010011)$ & 246 \\
$\mathcal{C}_{68,9}$ & $(1100101101101100010110010011011100)$ & 248 \\
$\mathcal{C}_{68,10}$ & $(0111011000111011001111101011000101)$ & 249 \\
$\mathcal{C}_{68,11}$ & $(1110010010000001100111101110001111)$ & 250 \\
$\mathcal{C}_{68,12}$ & $(1010001100110001100010101111011101)$ & 251 \\
$\mathcal{C}_{68,13}$ & $(1100000011010000110001001001110101)$ & 253 \\
$\mathcal{C}_{68,14}$ & $(0001110111001011010100110010010010)$ & 254 \\
$\mathcal{C}_{68,15}$ & $(1110100101000000011011110011010111)$ & 256 \\
$\mathcal{C}_{68,16}$ & $(0001110010100101110110011110000001)$ & 270 \\
$\mathcal{C}_{68,17}$ & $(1000111100000011111100010111100101)$ & 271 \\
$\mathcal{C}_{68,18}$ & $(0001001111010111110010101011110110)$ & 272 \\
$\mathcal{C}_{68,19}$ & $(1111111100001111110111100001110001)$ & 274 \\
$\mathcal{C}_{68,20}$ & $(0001111101000101010101001011100010)$ & 275 \\
$\mathcal{C}_{68,21}$ & $(1100011111100101010100100110101001)$ & 276 \\
$\mathcal{C}_{68,22}$ & $(1111001001111101100100101111111001)$ & 277 \\
$\mathcal{C}_{68,23}$ & $(1100100100001001101010111101110011)$ & 278 \\
$\mathcal{C}_{68,24}$ & $(1111100111011001111110100100111010)$ & 279 \\
$\mathcal{C}_{68,25}(101000110100111111110110100000010)$ & 281 \\
$\mathcal{C}_{68,26}$ & $(0011001100100101101010000110010111)$ & 282 \\
$\mathcal{C}_{68,27}$ & $(0011001010111110000111111010001111)$ & 283 \\
$\mathcal{C}_{68,29}$ & $(100110111110100011111001111000111)$ & 284 \\
& $(1100011101010011001101001010110110)$ & 289 \\
\hline
\end{tabular}

In Table 16, $\mathcal{C}_{68, i}=\left\langle\mathcal{D}_{7} \cap\langle x\rangle^{\perp}, x\right\rangle$ which is a neighbour of $\mathcal{D}_{7}$, that was obtained from the group $C_{33}$ in 4.6. Since first 34 columns of the generator matrix of $\mathcal{D}_{7}$ are linearly independent, without loss of generality we may assume that the first 34 entries of $x$ are 0 . 
Table 17: New codes of length 68 by Theorem 2.5

\begin{tabular}{ccccc}
\hline $\mathcal{C}_{68, i}$ & $\mathcal{C}_{i}$ & $\left(x_{17}, x_{18}, \ldots, x_{32}\right)$ & $\gamma$ & $\beta$ in $W_{68,2}$ \\
\hline $\mathcal{C}_{68,30}$ & $\mathcal{C}_{1}$ & $(u+1, u, 1, u+1, u, u+1,0,0, u, u, 1, u, u+1,1, u+1, u+1)$ & 1 & 51 \\
$\mathcal{C}_{68,31}$ & $\mathcal{C}_{1}$ & $(u, u, u, u+1,0,0,0, u+1, u, u, 1, u+1, u+1,1,1, u)$ & 1 & 53 \\
$\mathcal{C}_{68,32}$ & $\mathcal{C}_{1}$ & $(u+1,0,1,1,0, u+1,0, u, u, u, 1,0, u+1, u+1, u+1,1)$ & 1 & 55 \\
$\mathcal{C}_{68,33}$ & $\mathcal{C}_{3}$ & $(u, u, 1, u+1,0,1, u, u+1, u, u+1, u, u+1,1,1, u, 1)$ & 2 & 73 \\
$\mathcal{C}_{68,34}$ & $\mathcal{C}_{1}$ & $(1, u+1, u+1, u, u+1, u+1, u, 0, u+1,0, u, u, 0,0,1, u)$ & 2 & 75 \\
$\mathcal{C}_{68,35}$ & $\mathcal{C}_{1}$ & $(1,1,0,1, u+1, u, u+1,1,1, u+1,1, u+1,1,1,1,0)$ & 2 & 79 \\
$\mathcal{C}_{68,36}$ & $\mathcal{C}_{1}$ & $(u+1,1, u+1,0, u+1, u+1 u, u, u+1, u, u, 0, u, u, 1, u)$ & 2 & 83 \\
$\mathcal{C}_{68,37}$ & $\mathcal{C}_{3}$ & $(u+1,0,1, u+1,0,1, u, u, 0,0, u, 1,1,1,0, u)$ & 3 & 84 \\
$\mathcal{C}_{68,38}$ & $\mathcal{C}_{3}$ & $(0, u, u+1, u+1, u, 1, u, 1, u, u+1, u, u+1,1, u+1,0,1)$ & 3 & 95 \\
$\mathcal{C}_{68,39}$ & $\mathcal{C}_{1}$ & $(0,0, u, u+1, u+1, u+1, u, 1, u, 1,1, u+1, u+1, u+1,1,1)$ & 3 & 97 \\
$\mathcal{C}_{68,40}$ & $\mathcal{C}_{1}$ & $(0,0, u+1, u+1,0, u+1,0,1, u+1,0,1,0, u, u+1, u, u)$ & 3 & 109 \\
$\mathcal{C}_{68,41}$ & $\mathcal{C}_{1}$ & $(u+1,1,1,1,1,1,0,0,1, u+1,0, u+1, u, 0, u, 0)$ & 3 & 113 \\
\hline
\end{tabular}

Theorem 2.5 is applied over $\mathbb{F}_{2}+u \mathbb{F}_{2}$ for the codes in Table 17 . The $\mathcal{C}_{i}$ are the codes over $\mathbb{F}_{2}+u \mathbb{F}_{2}$ of length 32 obtained from groups of order 15, tabulated in Table 7 . When Theorem 2.5 is applied, we obtain self-dual codes over $\mathbb{F}_{2}+u \mathbb{F}_{2}$ of lengths 34 , whose binary images turn out to be extremal self-dual binary self-dual codes of length 68 .

We observe that the first 16 columns of $G$ are linearly independent. So, without loss of generality we assume that the first 16 entries of the extension vector $X$ are 0 , which narrows down the search field remarkably from $4^{32}$ to $4^{16}$.

\section{Conclusion}

The recent developments in using group rings to construct self-dual codes have extended the tools in the search for self-dual codes. The groups of different orders have introduced many new constructions that lead to different automorphism groups, which in turn fill the gap caused by the restrictive nature of constructions such as double circulant, bordered double circulant and four circulant constructions. The success of these new methods, which has been demonstrated in [13] and [12], is further shown to be the case for the methods we have introduced in this paper, which modify and extend the concept of bordered double circulant matrices as well as the classical group ring constructions that have been discussed in the aforementioned works. The remarkable number of extremal self-dual codes and the 41 new extremal self-dual codes of length 68 that have been obtained show the strength of 
the methods we have discussed.Due to computational limitations and page restrictions, we have considered a sample of groups in our constructions.

We propose two possible directions for future research. One is to look at different groups of different orders. However it should be noted that as the order of the group increases, so does the length of the codes obtained. This could potentially cause a computational problem for groups of higher order. The second possible line of research would be to apply the constructions to rings $R_{k}$ for $k \geq 2$. While we are confident of new results in this case, we would like to observe that, much like the groups of higher order, there will be a higher computational complexity when the constructions are applied to $R_{k}$ when $k \geq 2$. This is because $\left|R_{2}\right|=16,\left|R_{3}\right|=256$, etc.

Acknowledgement: We would like to thank the anonymous referees for their helpful comments and suggestions that have improved our paper.

\section{References}

[1] F. Bernhardt, P. Landrock, O. Manz, "The extended Golay codes considered as ideals", J. Comb. Theory, Ser. A, vol. 55, no. 2, pp. 235-246, 1990.

[2] W. Bosma, J. Cannon and C. Playoust, "The Magma algebra system. I. The user language", J. Symbolic Comput., vol. 24, pp. 235-265, 1997.

[3] S. Buyuklieva and I. Bouyukliev, "Extremal self-dual codes with an automorphism of order 2", IEEE Trans. Inform. Theory vol. 44, pp. 323-328, 1998.

[4] J.H. Conway and N.J.A. Sloane, "A new upper bound on the minimal distance of self-dual codes", IEEE Trans. Inform. Theory, vol. 36, no. 6, pp. 1319-1333, 1990.

[5] S.T. Dougherty, "Algebraic Coding Theory Over Finite Commutative Rings", SpringerBriefs in Mathematics. Springer, Cham, 2017, ISBN: 978-3-319-59805-5; 978-3-31959806-2.

[6] S.T. Dougherty, J. Gildea, R. Taylor and A. Tylshchak, "Group Rings, G-Codes and Constructions of Self-Dual and Formally Self-Dual Codes", Designs, Codes and Cryptography, 10.1007/s10623-017-0440-7.

[7] S.T. Dougherty, J.L. Kim, H. Kulosman and H. Liu, "Self-dual codes over commutative Frobenius rings", Finite Fields Appl., vol. 16, pp. 14-26, 2010.

[8] S.T. Dougherty, M. Harada and T.A. Gulliver, "Extremal Binary Self-dual Codes", IEEE Trans. Inform. Theory, vol. 43, no. 6, pp. 2036-2047, 1997. 
[9] S.T. Dougherty, P. Gaborit, M. Harada and P. Sole, "Type II codes over $\mathbb{F}_{2}+u \mathbb{F}_{2}$ ", IEEE Trans. Inform. Theory, vol. 45, pp. 32-45, 1999.

[10] S.T. Dougherty, B. Yildiz, and S. Karadeniz, "Codes over $R_{k}$, Gray maps and their Binary Images", Finite Fields Appl., vol. 17, no. 3, pp. 205-219, 2011.

[11] S.T. Dougherty, B. Yildiz, and S. Karadeniz, "Self-dual Codes over $R_{k}$ and Binary Self-Dual Codes", European Journal of Pure and Applied Mathematics, vol. 6, no. 1, pp. 89-106, 2013.

[12] S.T Dougherty, J. Gildea, A. Kaya and B. Yildiz, "New Self-dual and Formally Selfdual codes from Group Ring Constructions", submitted.

[13] J. Gildea, A. Kaya, R. Taylor and B. Yildiz, "Constructions for Self-dual Codes Induced from Group Rings", Finite Fields Appl., vol. 51, pp. 71-92, 2018.

[14] T.A. Gulliver and M. Haraada, "Classification of Extremal double circulant self-dual codes of lengths 74-88", Discrete Mathematics, vol. 306, no. 17, pp. 2064-2072, 2006.

[15] M. Harada and A. Munemasa, "Some restrictions on weight enumerators of singly even self-dual codes", IEEE Trans. Inform. Theory, vol. 52, pp. 1266-1269, 2006.

[16] T. Hurley, "Group Rings and Rings of Matrices", Int. Jour. Pure and Appl. Math, vol. 31, no. 3, pp. 319-335, 2006.

[17] T. Hurley, "Self-dual, dual-containing and related quantum codes from group rings", arXiv:0711.3983, 2007.

[18] S. Karadeniz and B. Yildiz, "Double-circulant and bordered-double-circulant constructions for self-dual codes over $R_{2}$ ", Advances in Mathematics of communications, vol. 6, no. 2, pp. 193-202, 2012.

[19] I. McLoughlin, "A group ring construction of the [48, 24, 12] Type II linear block code", Des. Codes Cryptogr., vol. 63, no. 1, pp. 29-41, 2012.

[20] I. McLoughlin and T. Hurley, "A group ring construction of the extended binary Golay code", IEEE Trans. Inform. Theory, vol. 54, no. 9, pp. 4381-4383, 2008.

[21] E.M. Rains, "Shadow Bounds for Self Dual Codes", IEEE Trans. Inf. Theory, vol.44, pp.134-139, 1998.

[22] M. Ventou and C. Rigoni, "Self-dual doubly circulant codes", Discrete Mathematics, vol. 56, no. 2-3, pp. 291-298, 1985.

[23] N. Yankov, M. Ivanova and M.H. Lee, "Self-dual codes with an automorphism of order 7 and s-extremal codes of length 68", Finite Fields Appl., vol. 51, pp. 17-30, 2018. 